\title{
RELACIÓN DEL SEXISMO EN LA SATISFACCIÓN MARITAL EN AREQUIPA METROPOLITANA
}

\section{Relationship between sexism and marital satisfaction in Metropolitan Arequipa}

\author{
Carolina Margarita Dianderas Cáceres* \\ Universidad Católica San Pablo
}

\begin{abstract}
Resumen
El propósito de este estudio fue definir la relación ente los tipos de sexismo ambivalente y la satisfacción marital. Del mismo modo se relacionó la influencia de variables sociodemográficas (edad, género y grado de instrucción) con los niveles de sexismo, con la finalidad de identificar posibles causas de la violencia de género en la ciudad de Arequipa. Para esto se aplicó un cuestionario con el inventario de reactivos del sexismo ambivalente de Glick y Fiske (1996) y de la satisfacción marital de Pick y Andrade (1988) a una muestra de cuatrocientas personas en la ciudad de Arequipa metropolitana, entre los 16 y 74 años. Los resultados mostraron la independencia de la satisfacción marital respecto a los niveles de sexismo ambivalente, pero confirmó la existencia de sexismo benévolo y hostil tanto en hombres como en mujeres, siendo estas últimas las que presentan los valores más altos de sexismo benevolente. También se halló que existe un mayor sexismo entre los jóvenes que en los adultos.
\end{abstract}

Palabras clave: sexismo, satisfacción marital, violencia de género, grado de instrucción.

\begin{abstract}
The purpose of this study was to define the relationship between types of ambivalent sexism and marital satisfaction. Likewise, the influence of socio-demographic variables (age, gender and educational level) was related to the levels of sexism in order to identify possible causes of gender violence in the city of Arequipa. For this, a questionnaire was applied with the inventory of reagents of the ambivalent sexism of Glick \& Fiske (1996) and of marital satisfaction of Pick $\&$ Andrade (1988) to a sample of four hundred people in the city of Arequipa metropolitan, between 16 and 74 years old. The results showed the independence of marital satisfaction from the levels of ambivalent sexism, but confirmed the existence of benevolent and hostile sexism in both men and women, the latter having the highest values of benevolent sexism. It was also found that there is more sexism among young people than in adults.
\end{abstract}

Keywords: sexism, marital satisfaction, gender violence, educational level.

\footnotetext{
* Psicóloga clínica. Docente investigador del Instituto para el Matrimonio y Familia de la Universidad Católica San Pablo.cmdianderas@ucsp.edu.pe
} 
Podemos definir el sexismo como actitudes discriminatorias hacia las personas en virtud de su pertenencia a un determinado sexo biológico. El sexismo ha estado presente y aun lo está en la sociedad contemporánea. La forma más común de mostrar sexismo es considerar a la mujer como inferior al hombre. Por años, la mujer siempre fue considerada un ser inferior al hombre y la edad moderna se inició con la pugna de las mujeres por alcanzar la igualdad.

Dentro de toda la gesta para reivindicar la igualdad de derechos de la mujer, para tratar de entender esta diferencia y superarla, se comenzaron a presentar estudios de género, los cuales han sido beneficiados por las aportaciones que han tenido desde los desarrollos de la llamada Psicología Social (Morales \& Moya, 2007). A partir del vínculo generado, los estudios de estereotipos se establecieron como un tema de relevancia para la Psicología Social en donde conceptos como liberación sexual y estereotipos opresores han sido muy relevantes; se enlazó la categoría de «masculinidad» a las ideas desarrolladas en torno a un concepto de patriarcado represivo (Morales \& Moya, 2007).

El devenir cultural ha continuado generando desarrollos en el campo de la Psicología, como los propuestos por los autores Glick y Fiske (1996) que establecen como propósito determinar los niveles de sexismo que habitualmente los varones exteriorizan respecto de las mujeres (García \& López, 2006), pudiendo ser este benévolo u hostil (Morales \& Moya, 2007). Esta preocupación por los estilos de vida y los estereotipos ha permitido reducir todas las diferencias entre los sexos a definiciones de roles entre hombres y mujeres, vinculadas a una idea de construcción que la persona hace de sí misma dentro de la sociedad que conforma, en donde el deber ser vinculado al hombre y la mujer se ha posicionado como un elemento que trastoca la equidad de género (Luengas \& Velandia, 2012).

De esta manera, para Glick y Fiske (1996) se entiende como sexismo hostil las actitudes tradicionales y prejuiciosas hacia las mujeres que suponen su inferioridad y se estructuran en tres categorías: a) un paternalismo dominador que considera a las mujeres débiles e inferiores y, por lotanto, necesitan ser dirigidas y controladas por el hombre; b) la diferencia de género competitiva que sostiene que las mujeres son diferentes a los hombres porque no poseen las características para triunfar en el ámbito público y por ello deben restringirse al ámbito privado; y c) la hostilidad heterosexual que alude al poder sexual que tienen las mujeres y que las hace peligrosas y manipuladoras para los hombres (Zubieta, Beramendi, \& Sosa, 2011). En contraposición, se entiende por sexismo benevolente al conjunto de actitudes interrelacionadas hacia las mujeres que son estereotipadas y se limitan a ciertos roles pero que producen un efecto positivo y suscitan en el perceptor conductas prosociales o de búsqueda de intimidad. Sus categorías son: a) paternalismo protector que implica el cuidado de la mujer por parte del hombre; b) diferenciación de género complementaria que enfatiza las características positivas de las mujeres que complementan a las de los hombres; y c) intimidad heterosexual que supone la dependencia diádica de los hombres con respecto a las mujeres para la reproducción y satisfacción sexual (Zubieta et al., 2011).

En paralelo, dentro del ámbito propio de la Psicología Social, autores como Pick y Andrade (1988) han procurado desarrollar un instrumento que persigue el propósito de determinar, a través de una escala, el grado de satisfacción marital que puede experimentar una pareja heterosexual que ha formado una comunidad de vida. La realidad marital es una dimensión de lo humano que se ha visto afectada por los cambios culturales y esos mismos fenómenos culturales ha propiciado su deformación, fuertemente vinculada a los desarrollos feministas que promueven, junto con una concepción andrógina de la sexualidad (Luna, 2003) una apología de la homosexualidad (Burggraf, 2010). Según Cortés, Reyes, Díaz-Loving, Rivera y Monjarrás (como se citó en de Lourdes, Calvo, \& De la Orta, 2011), el propósito de la Escala de Satisfacción Marital propone obtener la comprensión subjetiva de la persona en torno a su pareja y su relación. Pick y Andrade (como se citó en de Lourdes et al., 2011) han orientado sus opiniones a señalar que la satisfacción marital expresa «la actitud que un individuo tiene hacia su relación marital y hacía su cónyuge».

Por otro lado, Domínguez. (2012, p. 13) afirma que la satisfacción marital es «uno de los indicadores 
más extensamente estudiados de la felicidad y estabilidad de las relaciones». Dentro de la medición de la satisfacción marital, son múltiples los criterios que se han utilizado para poder determinar ese indicador: entre ellos, un criterio coincidente es el de la categoría antropológica de homogamia. El término anteriormente mencionado se refiere a la elección de una pareja similar a uno, con diversas características: religiosa, étnica, cultural, vivencial, etc. Dentro del abanico de escalas de satisfacción marital se ha decidido usar la escala de Pick y Andrade (1988), la cual considera que el concepto de satisfacción marital es útil para describir la actitud de un individuo respecto de su relación y hacia su cónyuge.

Desde el punto de vista psicosocial, al sexismo se le puede considerar como una de las principales causas que mantienen las desigualdades entre hombres y mujeres. En las culturas actuales, no se acepta el sexismo hostil que representa la forma más agresiva de discriminación de la mujer; sin embargo, el sexismo benevolente que, con un cariz más sutil, idealiza a las mujeres como esposas, madres, delicadas y hermosas, es comúnmente aceptado incluso por las mujeres que cumplen estos roles tradicionales, satisfaciendo sus necesidades afectivas y las de los varones (Garaigordobil \& Aliri, 2011).

La realidad social peruana - una mezcla de culturas diversas - se explica a partir de un grupo social nativa, cambiante en las diversas regiones. A través de los años esta estirpe se ha amalgamado con la colonización española, que trajo no solamente la ibérica sino también la africana (asentados en ciertas zonas geográficas). La asiática llegó como consecuencia del desplazamiento ocurrido al finalizar la Segunda Guerra Mundial. Es así como se ha configurado un panorama particular, multirracial y multicultural (Santos, 2002). La dominación española ha dejado un estigma social que se traduce en una especie de sociedad patriarcal, entendiendo el patriarcado como el tipo de sociedad en la que lo masculino posee una supremacía o dominio (Asiyanbola, 2005; Stacey, 1993), donde el hombre aparece como cabeza de familia y la mujer se presenta como su apoyo; a pesar de los años transcurridos, estas diferencias se siguen presentando. Arequipa, una de las ciudades más importantes del país y lugar de asentamiento de muchos colonos españoles - recordemos que las labores del campo eran realizadas por los hombres - es ejemplo de esta sociedad sexista.

Este estudio pretende encontrar los grados de sexismo que existen en la ciudad de Arequipa y analizar las relaciones entre el sexismo y la satisfacción marital con otras variables sociodemográficas (sexo, edad, estado civil y grado de instrucción).

Al no existir estudios previos acerca de la influencia del sexismo en la satisfacción marital en la ciudad de Arequipa, pretendemos estudiar las relaciones del sexismo con la satisfacción de las parejas encuestadas, así como si los niveles de sexismo pudieran tener relación alguna con la edad, situación civil o grado de instrucción.

\section{METODOLOGÍA Y PARTICIPANTES}

Se realizó un estudio descriptivo, correlacional, transversal (Hernández, Fernandez y Baptista).

La muestra fue de 400 personas de la ciudad de Arequipa. La selección fue no probabilística y por cuotas en función de la residencia en cuatro distritos de Arequipa metropolitana.

La muestra un 55\% eran mujeres (220) y el $45 \%$ hombres (180) - fue extraída proporcionalmente de los siguientes distritos: Cerro Colorado, Cayma, Sachaca y José Luis Bustamante y Rivero. La edad fluctúa entre 16 y 74 años, siendo la media de 40,1 años. Un $65,5 \%$ respondió ser casado, el 33,8\% conviviente, el $0,5 \%$ lo componían divorciados y solamente el 0,3 $\%$ solteros. De la muestra el $47 \%$ reportó tener un grado de instrucción superior (universitario), el 32,5 $\%$ instrucción secundaria, el $18 \%$ instrucción técnica y el 2,5\% completaron únicamente la Educación Primaria.

\section{INSTRUMENTOS}

\section{Inventario de sexismo ambivalente}

Esta prueba fue creada originalmente por Glick y Fiske en 1996 y fue adaptada al español por Espósito, Moya y Glick en 1998. Consta de 22 ítems y seis 
alternativas de respuesta en una escala tipo Likert de 0 a 5. Tiene una estructura factorial de cuatro dimensiones: sexismo hostil, sexismo benévolo (paternalismo protector), sexismo benévolo (diferenciación de género) y sexismo benévolo (intimidad heterosexual). Cuenta con niveles de confiabilidad adecuados alfa de Cronbach.

\section{Escala de satisfacción marital}

Elaborada por Pick y Andrade en 1988 para poblaciones latinoamericanas. Se trata de una prueba unidimensional que consta de 24 ítems con tres niveles de respuesta: 1 (me gusta cómo está pasando), 2 (me gustaría algo diferentes), 3 (me gustaría muy diferente). Cuenta con niveles de confiabilidad adecuados alfa de Cronbach.

El instrumento diseñado por Pick y Andrade está compuesto por tres dimensiones:

- Satisfacción en la interacción marital: evalúa la percepción de los cónyuges respecto a la satisfacción percibida en el matrimonio, comparándola con otros parámetros conocidos, como los de la familia y los miembros del círculo social más amplio.

- Satisfacción con los aspectos emocionales del cónyuge: satisfacción del cónyuge respecto a cómo se dan las experiencias afectivas y se muestran las emociones en su matrimonio.
- Satisfacción en los aspectos estructurales y la organización física del cónyuge: evalúa la satisfacción de los cónyuges en cuanto a cómo la pareja se organiza internamente y la forma en la que se enfrentan a su matrimonio (Domínguez, 2011, p. 21).

\section{Ficha de datos personales}

Se empleará además una ficha de recolección de datos personales que incluye los siguientes datos: sexo, edad, estado civil, número de hijos, grado de instrucción, ocupación, cargo, tiempo que tiene con la pareja.

\section{ANÁLISIS DE DATOS}

Se analizaron las variables utilizando estadística descriptiva. Asimismo para correlacionar las variables utilizamos el coeficiente de Pearson. Para todo el procesamiento estadístico empleamos el software SPSS 24 (IBM, 2016).

\section{RESULTADOS Y DISCUSIÓN DE RESULTADOS}

En la tabla 1, los valores de asimetría y curtosis están dentro de los rangos que indican que las variables se acercan a ser simétricas y mesocúrticas, por lo que los datos se han procesado con estadística paramétrica.

Tabla 1

Estadísticos descriptivos de las dimensiones de sexismo ambivalente

\begin{tabular}{lccccccr}
\hline & $N$ & Minimo & Máximo & Media & Desv. tip. Asimetria & Curtosis \\
\hline Sexismo hostil & 400 & 10 & 52 & 34,06 & 7,831 &,- 071 &,- 667 \\
Sexismo benevolente & 400 & 11 & 55 & 35,60 & 7,468 &,- 158 &,- 695 \\
Satisfacción marital & 400 & 24 & 66 & 47,30 & 8,845 &,- 395 &,- 016 \\
N válido (según lista) & 400 & & & & & & \\
\hline \hline
\end{tabular}


No se ha encontrado ninguna evidencia que confirme que la satisfacción marital tenga relación con los tipos de sexismo analizados, es decir, en Arequipa los índices de satisfacción marital no se relacionan ni con el sexismo hostil ni con el sexismo benevolente.
Tal como se puede apreciar en la tabla 2, la satisfacción marital no se asocia el sexismo hostil con $\mathrm{r}=0.042 \mathrm{y}$ $\mathrm{p}>0.05$ ni el sexismo benevolente con $\mathrm{r}=0.057$ y $\mathrm{p}$ $>0.05$.

Tabla 2

Correlaciones entre sexismo ambivalente y satisfacción marital

\begin{tabular}{|c|c|c|c|c|}
\hline & & Sexismo hostil & $\begin{array}{c}\text { Sexismo } \\
\text { benevolente }\end{array}$ & $\begin{array}{c}\text { Satisfacción } \\
\text { marital }\end{array}$ \\
\hline \multirow[t]{3}{*}{ Sexismo hostil } & Correlación de Pearson & 1 & $.655^{\prime}$ & .042 \\
\hline & Sig. (bilateral) & &, 000 & .397 \\
\hline & $\mathrm{N}$ & 400 & 400 & 400 \\
\hline \multirow[t]{3}{*}{ Sexismo benevolente } & Correlación de Pearson & & 1 & -.057 \\
\hline & Sig. (bilateral) & & & .254 \\
\hline & $\mathrm{N}$ & & 400 & 400 \\
\hline \multirow[t]{3}{*}{ Satisfacción marital } & Correlación de Pearson & & & 1 \\
\hline & Sig. (bilateral) & & & \\
\hline & $\mathrm{N}$ & & & 400 \\
\hline
\end{tabular}

En la tabla 3 se aprecia que existe una diferencia significativa entre los niveles de sexismo benevolente entre varones y mujeres. $(\mathrm{t}=-2,095 ; \mathrm{p}<0,05)$ siendo en las mujeres ligeramente más alto, pero no existe diferencia significativa entre sexismo hostil $(t=0,098$; $\mathrm{p}>0,05)$ y satisfacción marital entre varones y mujeres $(\mathrm{t}=0,824 ; \mathrm{p}>0,05)$, lo que contrasta con estudios previos como los de Feather y Boeckman (2007), Fowers y Fowers (2010), Garaigordi y Aliri (2011), Glick y Fiske (1996), Lameiras y Rodríguez (2003), Masser y Abrams (1999), Travaglia et al. (2009) que indican que existen evidencias de mayor sexismo hostil y benevolente en los varones. Agreguemos también los estudios de Chen, Fiske y Lee (2009) y Lameiras, Rodríguez, Calado, Foltz y González (2006) que resaltan que no hay diferencias en el sexismo benevolente con el género. El resultado de un mayor sexismo benevolente en mujeres concuerda con el estudio de Glick y Fiske (2000), donde en una muestra de hombres y mujeres en Chile resaltó que también en las mujeres el sexismo benevolente era alto puesto que las mujeres mismas tenderían a avalarlo (Cárdenas, Lay, González, Calderón, \& Alegría, 2010). Esto debido a que han sido criadas en sociedades históricamente patriarcales o machistas como la chilena o la peruana, donde la mujer es considerada «el sexo débil». Lo cual indicaría que este tipo de sexismo podría continuar en el futuro, lejos de disminuir, debido a las propias mujeres que lo aceptan deliberadamente para evitar otro tipo de situaciones sexistas más graves o intolerables (Cárdenas et al., 2010). Por tanto nos confirma que también en la muestra de nuestro análisis las mujeres aceptan la situación de sexismo o de patriarcado en la que se desenvuelven (Abrams, Viki, Masser, \& Bohner, 2003; Expósito, Moya, \& Glick, 1998), lo que nos lleva a presumir que el «predominio» de los varones sobre las mujeres es una constante en las distintas sociedades, y ese privilegio de los varones tiende a continuar este tipo de comportamiento de discriminación «social» contra las mujeres (Cruz, Zempoaltecatl, \& Correa, 2005). 
Tabla 3

Estadísticos descriptivos de Sexismo ambivalente de acuerdo al sexo

\begin{tabular}{llcccccr}
\hline \hline & Sexo & N & Media & Desviación tip. & t & gl & Sig. (bilateral) \\
\hline Sexismo hostil & Masculino & 180 & 34,10 & 7,703 & .098 & 398 & .922 \\
& Femenino & 220 & 34,02 & 7,952 & & & \\
\hline Sexismo benevolente & Masculino & 180 & 34,74 & 7,589 & $-2,095$ & 398 &, 037 \\
& Femenino & 220 & 36,30 & 7,309 & & &, 410 \\
\hline \multirow{2}{*}{ Satisfacción marital } & Masculino & 180 & 47,71 & 8,867 & \multirow{2}{*}{824} & 398 & \\
& Femenino & 220 & 46,97 & 8,833 & & & \\
\hline \hline
\end{tabular}

Sobre el caso de los hombres nuestro estudio, como otros previos (Cárdenas et al, 2010), constata que ellos usualmente presentan tanto el sexismo hostil como el benevolente. Lo cual podría interpretarse como que ellos estarían interesados en que se mantengan estos roles de género tradicionales para favorecer la continuidad de esta estructura patriarcal de la sociedad (Rottenbacher, 2010).

Sobre el estado civil, los resultados de la muestra tampoco arrojan indicios de relación alguna entre el sexismo benevolente, sexismo hostil o satisfacción marital en función de si es soltero, casado, conviviente o divorciado.

Respecto a la edad de los entrevistados en nuestra muestra de cuatrocientas personas - en la que la mínima fue de 16, la máxima de 74 y con una media de 40 años - los resultados nos confirman que la edad sí tiene una relación significativa tanto con el sexismo hostil como con el sexismo benevolente, de modo que a mayor edad menor sexismo y a menor edad mayor sexismo (ver tabla 4). Este resultado es semejante a otros estudios (Fernández et al, 2006 ; Zakrisson, Anderzén, Lenell, \& Sandelin, 2012), que muestran que los adolescentes tienen mayor sexismo - hostil y benevolente - que los adultos. Un estudio que incluye edades desde la adolescencia hasta los 70 años confirma que existe sexismo benevolente y hostil, con valores altos, desde la adolescencia; este se reduce en la edad adulta hasta los 54 años; entre los 64 y 70 el sexismo aumenta hasta un valor máximo

Tabla 4

Correlaciones sexismo ambivalente y edad

\begin{tabular}{llrrr}
\hline \hline & & $\begin{array}{c}\text { Sexismo } \\
\text { hostil }\end{array}$ & $\begin{array}{c}\text { Sexismo } \\
\text { benevolente }\end{array}$ & $\begin{array}{c}\text { Satisfacción } \\
\text { marital }\end{array}$ \\
\hline Edad & Correlación de Pearson &,$- 137^{* *}$ &,$- 108^{\circ}$ &,- 068 \\
& Sig. (bilateral) &, 006 &, 031 &, 173 \\
& $\mathrm{~N}$ & 400 & 400 & 400 \\
\hline
\end{tabular}

"Correlación significativa al nivel 0.05 (bilateral)

"Correlación significativa al nivel 0.01 (bilateral) 
Respecto al nivel socioeconómico, el análisis demuestra que no existe relación de este con los tipos de sexismo benevolente y hostil ni con la satisfacción marital. Esto en contraposición con otros estudios que muestran que los niveles socioeconómicos bajos evidencian menores niveles de sexismo hostil y benévolo que los niveles económicos más altos (Cárdenas et al., 2010).

Analizando el nivel de satisfacción encontrado en la muestra realizada, las personas con instrucción primaria o técnica presentan niveles de satisfacción marital medios o altos, alrededor del $80 \%$. También se aprecia que la satisfacción marital alta se tiene en entrevistados con instrucción primaria y que disminuye en la medida en que aumenta el grado de instrucción. Este resultado es comparable con otros estudios (Domínguez, 2011; García, Fuentes, \& Sánchez, 2016). Sin embargo, no es posible relacionar la influencia del grado de instrucción como una influencia decisiva en la satisfacción marital en la ciudad de Arequipa.

El objetivo principal de la presente investigación era determinar la relación entre la satisfacción marital con los grados de sexismo en los habitantes de la ciudad de Arequipa, y aunque esto no ha podido comprobarse se han encontrado hallazgos importantes que discutimos a continuación.

Los resultados - tanto para hombres como para mujeres - respecto del sexismo benevolente y hostil evidencian que no existen diferencias significativas según el grado de instrucción, esto en contraposición con otro estudio previo (Cruz et al., 2005). Incluso en este estudio, el sexismo benevolente se presenta en las mujeres con una relación estadística más fuerte, que podría explicar cómo las mujeres de bajo nivel educativo son más propensas a aceptar el sexismo y la sumisión por no disponer de recursos para enfrentarlo. Sin embargo, nuestro estudio nos indica que en Arequipa mujeres y hombres presentan sexismo hostil y benevolente independiente de su nivel de estudios; otro estudio (Garaigordobil \& Aliri, 2011) confirma que en las mujeres el nivel de instrucción no es predictor de su nivel de sexismo benevolente. Esto demanda reflexionar pues los esfuerzos de mejora en los niveles de educación podrían no tener un efecto positivo en la disminución del sexismo ambivalente que se presenta en los habitantes de la ciudad de Arequipa.

Una situación similar podemos apreciar con la satisfacción marital, que se presenta tanto en varones $\mathrm{y}$ mujeres con total independencia del nivel de instrucción.

En la muestra se ha encontrado que existe sexismo hostil y benevolente en los hombres de Arequipa, se requeriría de estudios sociodemográficos más detallados que permitan indagar las causas de la elevada violencia contra las mujeres, especialmente de sus parejas. Estudios previos (Abrams et al., 2003; Cruz et al., 2005) evidencian que existe cierta relación entre la violencia de género y la existencia tanto de sexismo hostil como benevolente. Resalta también, y aunque no es el objetivo de esta investigación, lo encontrado acerca de que la juventud se presenta más sexista que los adultos promedio, como también se puede constatar en investigaciones previas (Rudman $\&$ Phelan, 2008; Diaz-Aguado, 2003).

Ahora bien, otras investigaciones han encontrado relación entre los tipos de sexismo con conductas agresivas (García, Palacios, Torrico, \& Navarro, 2009; Herrera, Expósito, \& Moya, 2012; Yamawaki, Ostenson, \& Brown, 2009). Convendría realizar investigaciones focalizadas para evaluar las razones del alto nivel de sexismo de los jóvenes y qué tanto podrían estar relacionadas con la violencia hacia las mujeres en la ciudad de Arequipa.

Estudios previos también evidencian la relación del sexismo con la violencia de género (Garaigordobil \& Aliri, 2011) y sobre todo en la adolescencia (Diaz-Aguado, 2003). Sería muy recomendable la implementación de programas de educación entre los adolescentes para prevenir la violencia de género, ya que Arequipa es la segunda ciudad del Perú con los más altos índices de violencia contra las mujeres pues en el año 2015 se presentaron nueve casos de feminicidio, después de Lima que presentó veintisiete casos el mismo año (INEI, 2016, NP $N^{\circ}$ 116). Estos programas deberían enfocarse en la promoción de ambientes en los que se fomente la igualdad entre los sexos y se evite contextos educativos donde exista el racismo o actitudes prejuiciosas. 


\section{CONCLUSIONES}

Si bien no se ha encontrado relación entre la satisfacción marital con los tipos de sexismo hostil o benevolente, se ha determinado que el sexismo en Arequipa no solo se presenta en los hombres sino también en las mujeres, las cuales tienen sexismo benevolente en nivel medio-alto, esto confirma el tipo de sociedad tradicionalista de Arequipa en la que la mujer acepta y aprueba los roles tradicionales, el hogar, la crianza de los hijos, etc., y en la que los hombres pretenderían mantener ese mismo tipo de sociedad.

Las evidencias muestran que existiría una relación entre la violencia de género con los niveles de sexismo $\mathrm{y}$, particularmente, con los niveles de sexismo en la adolescencia. Se presenta la oportunidad de diseñar e implementar programas de educación y capacitación dirigidos a los jóvenes, especialmente en edad escolar, para proporcionarles conocimientos no solo en temas sexuales sino sobre todo en temas de afectividad, de modo que coadyuve a la persona en su maduración que es tan importante durante la adolescencia. Esto junto con programas dirigidos a jóvenes en los cuales se promueva la convivencia igualitaria donde no exista ningún tipo de racismo, para evitar situaciones de prejuicios y se fomente la igualdad entre todos, una igualdad intercultural y racial.

\section{REFERENCIAS}

Abrams, D., Viki, G. T., Masser, B., \& Bohner, G. (2003). Perceptions of stranger and acquaintance rape: the role of benevolent and hostile sexism in victim blame and rape proclivity. Journal of personality and social psychology, 84(1), 111125.

Burggraf, J. (2010). Varón y mujer: ¿naturaleza o cultura? El tratamiento del Género en la escuela, I Congreso Internacional sobre Educación Diferenciada, Ponencias y Comunicaciones, 1536.

Cárdenas M., Lay, S. L., González C., Calderón C., Alegría I. (2010). Inventario de sexismo ambivalente: Adaptación, validación y relación con variables psicosociales. Salud y Sociedad, l(2), 125-135.
Chen, Z., Fiske, S., \& Lee, T. (2009). Ambivalent sexism and power-related gender-role ideology in marriage. Sex Roles, 60, 765-778.

Cruz, C. E., Zempoaltecatl, V., \& Correa, F. E. (2005). Perfiles de sexismo en la ciudad de México: validación del cuestionario de medición del sexismo ambivalente. Enseñanza e Investigación en Psicología, 10(2), 381-395.

De Lourdes, Eguiluz L., Calvo, R. M., \& De la Orta, D. (2012). Relación entre la percepción de la satisfacción marital, sexual y la comunicación en parejas. Revista Peruana de Psicología y Trabajo Social, 1, 15-28.

Diaz-Aguado, M. J. (2003). Adolescencia, sexismo y violencia de género. Papeles del Psicólogo, 84, $35-44$.

Domínguez Domínguez, I. (2011). Influencia de la familia en la sexualidad adolescente. Revista Cubana de Obstetricia y Ginecología, 37(3), 387398.

Domínguez, E. (2012). Estudio sobre satisfacción marital y variables asociadas en parejas españolas. TFM. Universidad de Salamanca.

Expósito, F., Moya M., \& Glick, P. (1998). Sexismo ambivalente: medición y correlatos. Revista de Psicología Social, 13(2), 159-169.

Fernández, M. L., Castro, Y. R., Otero, M. C., Foltz, M. L., \& Lorenzo, M. G. (2006). Sexism, vocational goals, and motivation as predictors of men's and women's career choice. Sex Roles, 55(3-4), 267272.

Feather N. T., \& Boeckmann, R. (2007). Beliefs about gender discrimination in the workplace in the context of affirmative action: Effects of gender and ambivalent attitudes in an Australian sample. Sex Roles, 57(1-2), 31-42.

Fowers, A., \& Fowers, B. (2010). Social dominance and sexual self-schema as moderators of sexist reactions to female subtypes. Sex Roles, 62(7-8), 468-480.

Galicia, F. (1989). Una investigación sobre la escala de satisfacción marital. Revista Latinoamericana de Psicología, 21(3), 423-436. 
Garaigordobil, M. (2013). Sexismo y apego inseguro en la relación de pareja. Revista Mexicana de Psicología, 30(1), 53-60.

Garaigordobil, M., \& Aliri, J. (2011). Sexismo hostil y benevolente: relaciones con el autoconcepto, el racismo y la sensibilidad intercultural. Revista de Psicodidáctica, 16(2), 331-350.

Garaigordobil M., \& Aliri, J. (2013). Ambivalent sexism inventory: standardization and normative data in a sample of the Basque Country. Behavioral Psychology / Psicología Conductual, 21(1), 173186.

García, F. E., Fuentes, R., \& Sánchez, A. (2016).Amor, satisfacción en la pareja y resolución de conflictos en adultos jóvenes. Ајаyu. Órgano de Difusión Científica del Departamento de Psicología de la Universidad Católica Boliviana «San Pablo», 14(2).

García, R. \& López, E. (2006). Congruencia del rol de género y liderazgo: el papel de las atribuciones causales sobre el éxito y el fracaso. Revista Latinoamericana de Psicología, 38(2), 245- 257.

García, P., Palacios, M. S., Torrico, E., \& Navarro, Y. (2009). El sexismo ambivalente: ¿un predictor de maltrato? Asociación Latinoamericana de Psicología Jurídica y Forense.

George, P. (2009). Moral Pública. Chile: Instituto de estudios de la sociedad.

Glick, P. (2000). Beyond prejudice as simple antipathy: hostile and benevolent sexism across cultures. Journal of personality and social psychology, 79(5), 763-775.

Glick, P. \& Fiske, S., (1996). The Ambivalent sexism inventory: Differentiating hostile and benevolent sexism. Journal of Personality and Social Psychology, 70, 491-512.

Glick, P., Fiske, S. T., Mladinic, A., Saiz, J. L., Abrams D., \& Masser et al. (2000). Beyond prejudice as simple antipathy: hostil and benevolent sexism across cultures. Journal of Personality and Social Psychology, 79, 763-775.

Gómez, J., Apodaca, P., Ortiz, M. J. (2002). Apego y satisfacción afectivo-sexual en la pareja. Psicothema, 14, 469-475.
Guzmán, M., \& Contreras, P. (2012). Estilos de apego en relaciones de pareja y su asociación con la satisfacción marital. Psykhe, 21(1), 69-82.

Hadjadj, F. (2008). La Profundidad de los Sexos. Por una Mística de la Carne. España: Nuevo inicio.

Herrera, M. C., Expósito, F., \& Moya, M. (2012). Negative reactions of men to the loss of power in gender relations: Lilith vs. Eve. The European Journal of Psychology Applied to the Legal Context, 4(1), 17-42.

Hubeñák, F., Gómez, G., \& Santiago M. E. (2007). Historia integral de Occidente desde una perspectiva cristiana. Argentina: Educa.

Instituto Nacional de Estadística e Informática del Perú. (Junio, 2016). Nota de Prensa N. ${ }^{\circ} 116$.

Juan Pablo II. (1988). Carta Apostólica Mulieris Dignitatem del sumo pontífice Juan Pablo II sobre la dignidad y la vocación de la mujer con ocasión del año mariano.

Luengas, H. T., \& Velandia, A. (2012). Nivel de sexismo en consumidores y su valoración sexista de la publicidad. Suma Psicológica, 19(2), 75-88.

Luna, I. (2003). Androginia y postmodernismo: Una aproximación desde la Psiquiatría. Recuperado de https://www.psiquiatria.com/trastornos_ sexuales/androginia-y-postmodernismo-unaaproximacion-desde-la-psiquiatria/

Masser, B., \& Abrams, D. (1999). Contemporary sexism. The relationship among hostility, benevolence and neosexism. Psychology of Women Quarterly, 23, 503-517.

Morales, J. F. (2007). Definición de Psicología Social. En J. F. Morales, M. C. Moya, E. Gaviria e I. Cuadrado (Coords.), Psicología Social ( $3^{\mathrm{a}}$ ed.). Madrid, España: McGraw-Hill.

Pick de Weiss, S., \& Andrade, P. (1988). Desarrollo y validación de la Escala de Satisfacción Marital. Psiquiatría, 4(1), 9-20.

Rottenbacher de Rojas, J. M. (2010). Sexismo ambivalente, paternalismo masculino e ideología política en adultos jóvenes de la ciudad de Lima. Pensamiento psicológico, 7(14). 
Rudman L. A., \& Phelan, J. E. (2008). Backlash effects for disconfirming gender stereotypes in organizations. En A. P. Brief y B. M. Staw (Eds.), Research in organizational behavior, 4, 61-79. New York, NY: Elsevier.

Santos, M. (2002). La «cuestión racial»: un ajuste de cuentas en tiempos de globalización y postmodernidad. Debates en sociología, (27), 133-171.

Stacey, J. (1993). Untangling feminist theory. En D. Richardson y V. Robinson (Eds.), Introducing Women's Studies: Feminist Theory and Practice, 49-73. London: Macmillan.

Stein, E. (2007). La Estructura de la Persona Humana ( $3^{\mathrm{a}}$ ed.). España: Biblioteca de Autores Cristianos.

Travaglia, L. K., Overall, N. C., \& Sibley, C. G. (2009). Benevolent and hostile sexism and preferences for romantic partners. Personality and Individual Differences, 47, 599-604.
Wojtyla, K. (2009). Amor y Responsabilidad (4a ed.). España: Biblioteca Palabra.

Yamawaki, N., Ostenson, J., \& Brown, C. R. (2009). The functions of gender role traditionality, ambivalent sexism, injury, and frequency of assault on domestic violence perception: A study between Japanese and American college students. Violence Against Women, 15, 1126-1142.

Zakrisson I., Anderzén M., Lenell, F., \& Sandelin, H. (2012). Ambivalent sexism: A tool for understanding and improving gender relations in organizations. Scandinavian Journal of Psychology, 53(1), 64-70.

Zubieta, E., Beramendi, M., \& Sosa, F. (2011). Sexismo ambivalente, estereotipos y valores en el ámbito militar. Revista de Psicología (PUCP), 29(1), 101-130.

Fecha de recepción: 18 de octubre, 2017

Fecha de aceptación: 17 de noviembre, 2017 